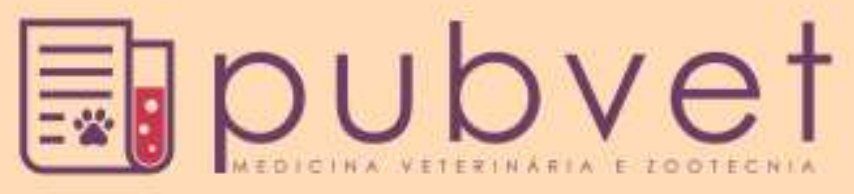

HTTP://DX.DOI.ORG/10.22256/PUBVET.V11N3.281-284

\title{
A importância dos endoparasiticidas e ectoparasiticidas em animais domésticos: Revisão
}

\author{
Iris Moniele Santos de Oliveira ${ }^{1 *}$, Islan Barbosa do Carmoํㅗ João Hugo Silveira Cruz ${ }^{1}$, \\ Maisa Jesus dos Santos', Leonardo Alves de Farias²
}

\begin{abstract}
${ }^{1}$ Discente do curso de Medicina Veterinária da Faculdade Pio Décimo do Estado de Sergipe. Aracaju - SE Brasil. ${ }^{2}$ Docente do curso de Medicina Veterinária da Faculdade Pio Décimo do Estado de Sergipe, Aracaju - SE Brasil. *Autor para correspondência, Endereço: Rua Milton Magalhães 74. Bairro: Ponto Novo- Loteamento Areias. CEP: 4909 7466. Cidade: Aracaju, SE. E-mail: miss_ellengata@hotmail.com
\end{abstract}

\begin{abstract}
RESUMO. As parasitoses gastrintestinais, entre elas as verminoses, são responsáveis por elevadas perdas econômicas, em decorrência do crescimento retardado, perda de peso, redução no consumo de alimentos, queda da produção de leite, baixa fertilidade e até mortalidade. O parasitismo é o maior flagelo que acomete os rebanhos mundiais. Os parasitos externos ocupam lugar de destaque entre as parasitoses dos animais domésticos. Os endoparasiticidas e ectoparaciticidas têm como função auxiliar na prevenção e combate as pragas, assim podendo diminuir o índice de certas doenças como, erliquiose e babesiose. A utilização profilática de endoparasiticidas e ectoparasiticidas em animais de companhia assume uma importância fundamental em nível de Saúde Animal, mas também no que respeita à Saúde Pública e Ambiental, face ao potencial zoonótico de determinados parasitas. A aplicação adequada e regular de antiparasitários recai sobre os proprietários para uma proteção contínua do animal, contra qualquer infecção parasitária. Nos carnívoros domésticos, cães e gatos, o maior desafio é o controle das pulgas e da sarna demodécica.
\end{abstract}

Palavras chave: Ectoparasiticidas, endoparasiticidas, saúde pública, zoonose

\section{The importance of endoparasiticides and ectoparasiticides in domestic animals: Review}

\begin{abstract}
Gastrointestinal parasites, including verminoses, are responsible for high economic losses, due to delayed growth, weight loss, reduction of food consumption, drop in milk production, low fertility and even mortality. Parasitism is undoubtedly the greatest scourge that affects the world's herds. The external parasites occupy a prominent place among the parasites of domestic animals. Endoparasiticides and ectoparaciticidas have as an auxiliary function in the prevention and combat of pests, thus reducing the rate of certain diseases, such as ehrlichiosis and babesiosis. Prophylactic use of endoparasiticides and ectoparasiticides In pet animals is of fundamental importance at the level of Animal Health, but also with regard to Public and Environmental Health, given the zoonotic potential of certain parasites. The proper and regular application of antiparasitics falls on the owners for a continuous protection of the animal, against any parasitic infection. In domestic carnivores, dogs and cats, the biggest challenge is controlling fleas and demodectic mange.
\end{abstract}

Keywords: ectoparasiticides, endoparasiticides, publichealth, zoonoses

\section{La importancia de endoparasiticidas y ectoparasiticidas de los animales domésticos: Revisión}


RESUMEN. Las parasitosis gastrointestinales, entre ellas las verminosas, son responsables por grandes pérdidas económicas, como resultado del retraso de crecimiento, pérdida de peso, reducción en el consumo de alimentos, disminución de la producción de leche, baja fertilidad e incluso mortalidad. El parasitismo es el mayor flagelo que afecta a los rebaños mundiales. Los parásitos externos ocupan un lugar destacado entre los parásitos de los animales domésticos. Los endoparasiticidas y ectoparaciticidas tienen la función de ayudar a prevenir y combatir las plagas, y pueden disminuir el indice de ciertas enfermedades como la erlichiosis y la babesiosis. El uso profiláctico de endoparasiticidas y ectoparasiticidas de los animales de compañía es de fundamental importancia en el nivel de salud animal, mas también respecto a la salud pública y al medio ambiente, dado el potencial zoonótico de ciertos parásitos. La aplicación adecuada y regular de antiparasitario recae sobre los propietarios para la protección continua del animal, contra cualquier infección parasitaria. En los carnívoros domésticos, perros y gatos, el mayor desafío es el control de pulgas y sarna.

Palabras clave: Ectoparasiticidas, endoparasiticidas, salud pública, zoonosis

\section{Introdução}

Protozoários, artrópodes e helmintos são considerados os principais causadores de morbimortalidade de seres humanos. A malaria, ocasionada pelo Plasmodium afeta aproximadamente 250 milhões de pessoas, enquanto um terço da população humana alberga helmintos intestinais como Ascaris lumbricóidese (Andrade et al., 2002).

Parasiticida é um veneno mais tóxico aos parasitas do que aos hospedeiros. Algumas vezes, o grau de descriminação é pequeno, outras, considerável, porém nunca completo, de forma que aplicação de parasiticidas sempre envolve algum risco ao hospedeiro. Claramente, algumas vezes é mais fácil explicar os efeitos deletérios que os parasiticidas frequentemente exercem sobre o hospedeiro do que explicar como eles atam os parasitas (Bowman, 2010).

O contato próximo do homem com cães e gatos como companhia verdadeira numa população humana envelhecendo e cada vez mais imunocomprometida tem aumentado a importância e a probabilidade de transmissão de infecções parasitárias com potencial zoonótico (Maddison et al., 2011).

A necessidade de combater esses organismos vem de períodos remotos, com a utilização de produtos administrados de forma empírica, como é o caso do alho, hortelã e semente de abobora. Nos últimos 40 anos, desde o emprego do tiabendaloze em1961, a indústria farmacêutica veterinária tem avançado na descoberta de novos princípios ativos com grande espectro de ação e margem de segurança para os mamíferos. Atualmente, a linha de antiparasitários compreende a maior fatia do mercado brasileiro de produtos veterinários, com aproximadamente $35 \%$ de participação das classes terapêuticas (Andrade et al., 2002).

\section{Revisão de Literatura}

Os principais ectoparasitas que atuam em animais domésticos são ácaros, moscas, carrapatos, pulgas, piolhos, bernes e larvas. Vários quimioterápicos são utilizados no controle de ectoparasitas que incluem o grupo dos pesticidas, praguicidas ou defensivos agrícolas (utilizados na agricultura) e inseticidas (usados na veterinária e em instalações rurais e urbanas) (Andrade et al., 2002).

A introdução do tiabendazol (TBZ) no começo dos anos 60 marcou o início da era moderna dos anti-helmínticos de amplo espectro que fossem seguros e eficazes contra uma ampla variedade de parasitas e nematóides e poderiam ser administrado em esquemas versáteis (Reinemeyer, 1986).

Vários inseticidas disponíveis atualmente providenciam excelente eliminação da infestação de pulgas estabelecida nos cães e gatos, tais como: dinotefuran, fipronil, imidacloprid, metaflumizona, nitempiran, administrados oralmente eliminaram as pulgas dentro de 3 a 4 horas, enquanto formulações tópicas na forma de spot-on com efeito residual, contendo fipronil, imidacloprida ou selamectina demoram 12 a 48 horas (Merck, 2013).

As infecções parasitarias da pele respondem pela maior parte das doenças da pele dos pequenos animais. A hipersensibilidade por mordida de pulgas é de longe, a doença cutânea alérgica mais comum observada em cães e gatos (Wilkinson and Harvey, 1997). 
Os anti-helmínticos devem apresentar eficácia contra todos os estágios parasitados de todos os gêneros de helmintos, eliminando $100 \%$ dos trematódeos e cestódeos, para os quais não há resposta imunoprotetora do hospedeiro e pelo menos 95\% dos nematódeos (Andrade et al., 2002).

Os principais danos à saúde são: comprometimento na digestão e absorção dos alimentos, menor aproveitamento dos nutrientes, falta de apetite, perda de peso, fraqueza, pelos eriçados e sem brilho, aumento de volume e dor abdominal, vômitos e diarreia (Nelson and Couto, 2015).

Os animais podem se infectar pela ingestão de ovos, ingestão de água ou alimentos contaminados e/ou larvas de terceiro estágio no ambiente contaminado por fezes, penetração ativa de larvas através da pele do animal, ingestão de hospedeiros intermediários (pulgas, piolhos, roedores) contendo larvas dos vermes encistadas nos tecidos. Passagem de larvas da fêmea para os filhotes via: transplácentaria ou transmamária (Nelson and Couto, 2015).

Pulgas e carrapatos são extremamente resistentes, inclusive quando instaladas no ambiente em lugares como piso madeiras, carpetes. Devido à resistência desses ectoparasitas no ambiente, o tratamento somente dos cães não é eficaz para o combate e controle. Como parte de seu ciclo de vida ocorre no ambiente, este também deve ser tratado para combater os ectoparasitas indesejáveis (Nelson and Couto, 2015).

As populações de pulgas e carrapatos adultos que se encontram nos animais representam apenas $5 \%$ do total da população, os demais $95 \%$ estão no ambiente em forma de ovos ou larvas de pulgas; ou ovos, larvas ou ninfas de carrapato. Para controlar esses parasitas são necessários certos medicamentos e profilaxias: aplicações de ectoparasiticidas, uso de carrapaticidas: aplicar nos canis, casinha dos cães, em plantas e canteiros, atentando para frestas nas paredes ou pisos e ralos (Nelson and Couto, 2015).

Repetir o tratamento a cada 15 dias no caso de infestações muito severas ou 21 dias; devendo ser, no mínimo, três aplicações para interferir no ciclo reprodutivo e de desenvolvimento do parasita de forma eficiente (Nelson and Couto, 2015).

\section{Drogas utilizadas na medicina veterinária}

O tiabendazol foi usado extensamente em uma ampla gama de hospedeiros (ovinos, bovinos, caprinos,suínos, equinos, aves e humanos). O tiabendazol podia ser administrado em dose terapêutica única ou administrado de forma profilática em doses mais baixas no alimento por um período estendido. Alem de sua atividade de amplo espectro o tiabendazol possuem propriedades larvicidas e ovicidas (Reinemeyer, 1986).

A utilização de coleiras impregnadas com deltametrina a $4 \%$ mostrou-se eficaz tanto em estudos laboratoriais como em campo, causando queda na taxa de infecção em cães de áreas endêmicas, tanto por seu feito repelente, quanto por seu efeito inseticida, quando a utilizadas corretamente (Gontijo and Melo, 2004). Praziquantel prazinoisoquilona, anti-helmíntico para tratamento de infestações por cestóides. Pode ser tóxico para alguns peixes marinhos e espécies do gênero corydoras (Viana, 2014). No passado, foram utilizados vários medicamentos no tratamento de babesiose, mas apenas o aceturato de diminazeno e o dipropionato de imidocarbe ainda continuam sendo utilizados (Merck, 2013).

$\mathrm{O}$ tratamento das infestações por ectoparasitos deve levar em conta o ciclo biológico do parasito, seu potencial para contagio é a capacidade do parasito quanto a sobreviver no ambiente (Wilkinson and Harvey, 1997).

Permetrina piretróide ectoparasiticida é indicado para cães a partir de 4 semanas de idade na prevenção e controle das infestações por pulgas, carrapatos e mosquitos flebótomos.

Os parasitos exibem grande diversidade biológica, o que pode fazer com indivíduos capazes de tolerar efeitos de uma determinada dose anti-helmíntica continuem a resistência aos seus descendentes, enquanto seus parentes mais suscetíveis perecem frente ao mesmo produto (Andrade et al., 2002).

O amitraz diamida com atividade alfa ${ }^{2}$ adrenérgico ectoparasiticida e, experimentalmente, agente anestésico. Usar com cautela em diabéticos gestantes e cães com menos de quatro meses de idade. Cães de raças pequenas são mais sensíveis ao fármaco e devem receber apenas a metade da diluição. Não se deve usar em equíídeos e coelhos (Viana, 2014). 
A ivermectina constitui em um tratamento mensal profilático altamente eficaz; eficácia retroativa desde que quatro meses depois da infecção, se a administração mensal for continuada a por 12 meses; quando associada ao pamoato de pirantel (Heartgard Plus MerialInselin, NJ, USA) também controlada infecção por ancilostomídeos e nematoides; pode ser administrada com segurança a cães com microfilaremia,inclusive aos cães tidos como sensíveis a ivermectina (Barr, 2010).

O fipronil fenilpirazólico inibidor do GABA pulicida e carrapaticida, não deve ser utilizado em coelhos, pois sua ação pode ser fatal, em gatos com menos de 12 semanas de idade ou cachorros com menos de 10 semanas de idade, usar com cautela em idosos, gestantes e lactantes (Nelson and Couto, 2015).

\section{Considerações Finais}

Os endoparasiticidas e ectoparasiticidas são tratamentos auxiliares baseados no histórico clinico do animal é de suma importância. O sucesso e a eficácia no tratamento contra endoparasiticidas e ectoparasiticidas dependeram do comprometimento do tutor em parceria com o médico veterinário.

\section{Referências bibliográficas}

Andrade, S. F., Santarém, V. A. \& Andrade, S. F. 2002. Endoparasiticidas e ectoparasiticidas. Manual de terapêutica veterinária, 2, 437-476.

Barr, S.C \& Bowman, D.D. 2010. Dirofilariose Canina - Doenças Infecciosas e Parasitárias em cães e gatos - Consulta em 5 minutos. Rio de Janeiro, 182-183
Bowman, D. D. 2010. Parasitologia veterinária. Elsevier.

Gontijo, C. M. F. \& Melo, M. N. 2004. Leishmaniose visceral no Brasil: quadro atual, desfios e perspectivas. Revista Brasileira de Epidemiologia, 7, 338-349.

Maddison, J. E., Page, S. W. \& Church, D. B. 2011. Farmacologia clínica de pequenos animais. In: Philip, G. A. \& Thomas, A. F. (eds.) Medicamentos e reprodução. Elsevier Brasil, São Paulo.

Merck Manual de Veterinária: Hemoparasitos- $10^{\mathrm{a}}$ ed: São Paulo, Roca, 2013 p. 44-49.

Nelson, R. W. \& Couto, C. G. 2015. Medicina interna de pequenos animais. Elsevier Editora, Amsterdan.

Reinemeyer, C. R. 1986. Small strongyles. Recent advances. The Veterinary clinics of North America. Equine practice, 2, 281-312.

Viana, F. A. B. 2014. Guia terapêutico veterinário.

Wilkinson, G. T. \& Harvey, R. G. 1997. Atlas colorido de dermatologia dos pequenos animais: guia para o diagnóstico. Manole Ltda., São Paulo.

\section{Article History:}

Received 8 December 2016

Accepted 20 January 2017

Available on line 16 February 2017

License information: This is an open-access article distributed under the terms of the Creative Commons Attribution License 4.0, which permits unrestricted use, distribution, and reproduction in any medium, provided the original work is properly cited. 